\title{
ABOUT THE APPLICATIONS OF UNMIXING-BASED DENOISING FOR HYPERSPECTRAL DATA
}

\author{
Daniele Cerra, Rupert Müller, Peter Reinartz
}

German Aerospace Center (DLR)

Muenchner Strasse 20, Oberpfaffenhofen, 82234 Wessling, Germany

daniele.cerra@dlr.de, rupert.mueller@dlr.de, peter.reinartz@dlr.de

KEY WORDS: Hyperspectral remote sensing, denoising, spectral unmixing

\begin{abstract}
:
Unmixing-based Denoising is a recently defined method which exploits spectral unmixing to recover bands characterized by a low Signal-to-Noise Ratio in a hyperspectral scene. The output of the unmixing process, which aims at decomposing each image element in signals typically related to pure materials, is inferred into the pixelwise reconstruction of a given band, ignoring the residual vector which is mainly characterized by undesired atmospheric influences and sensor-induced noise. The reconstructed images exhibit both high visual quality and reduced spectral distortions. This paper analyses the main problems that must be taken into account when applying this technique to real data. Special attention is given to the reference spectra used in the linear mixing model, which should be selected in order to keep the informational content of a given band unaltered in the reconstruction step.
\end{abstract}

\section{INTRODUCTION}

The spectral range characterizing data acquired by state-of-theart hyperspectral sensors mostly spans the frequencies between $400 \mathrm{~nm}$ and $2500 \mathrm{~nm}$. Some bands are related to frequencies which are mostly absorbed by the atmosphere, such as the ones in the near-ultraviolet and blue portions of the spectrum. As the sensor receives a low energy signal at such frequencies, these are typically characterized by a low Signal to Noise Ratio (SNR). On the other hand, at other frequencies sensor-induced noise becomes predominant. As a consequence, these bands are often discarded in a preprocessing step common to most practical applications. For some tasks, it would be desirable to keep such spectral information to better estimate some specific parameters from the data.

Spectral unmixing (Bioucas-Dias et al., 2012) and denoising of hyperspectral images (Renard et al., 2008) have always been regarded as separate problems. By considering the physical properties of a mixed spectrum, Unmixing-based Denoising has been recentely introduced in (Cerra et al., 2013b) as a methodology representing any pixel as a linear combination of reference spectra in a hyperspectral scene. As the residual vector from the unmixing process is largely due to atmospheric interferences and instrument-induced noise, we can reconstruct each pixel ignoring the residual vector, and along with it most of the noise affecting each pixel.

As the quality of the denoised image will greatly depend on the adopted mixing model, distortions introduced by imperfections in the model should be kept to a minimum, and several problems arise when applying this technique to real data. To begin with, noise may be present in the very spectra used as a basis for spectral unmixing: to avoid this, spectra which are similar according to given criteria can be averaged to reduce noise influences. Furthermore, different samples of the same material may present subtle differences in terms of spectral response, which should be captured in the model. Finally, it is important to use a set of spectra which allows a complete representation of the informational content of a given band in a scene. This paper proposes an algorithm to tackle this problem by iteratively adding spectra to the model: these are chosen according to the distortions in the reconstructed image, whenever these deviate significantly from the typical random noise distribution. Experiments show that the proposed method can effectively retrieve information from corrupted bands characterized by a low SNR which are usually discarded, and could be useful to derive indices and parameters for specific applications.

The paper is structured as follows. Section 2 introduces Unmixingbased Denoising, while Section 3 illustrates the main challenges faced when using this algorithm in practical applications and how to tackle them. We conclude in Section 4.

\section{UNMIXING-BASED DENOISING}

The Unmixing-based Denoising (UBD) is a simple procedure which can be described as follows. Given a training dataset containing $n$ spectra, homogeneous to some degree, from each of $k$ materials, a set of reference spectral signatures is defined as $A=\left\{x_{1}, \ldots, x_{i}, \ldots, x_{k}\right\}$, where $x_{i}$ is the average of the $n$ spectra belonging to material $i$. Considering the mean value for a given reference spectrum reduces the presence of noise to a minimum, if each class is spectrally homogeneous. It must be remarked that no assumption on the purity of the reference spectra is made. Then, for each hyperspectral image element $m$ with $p$ bands, with $p<<k$, any unmixing procedure can be employed to decompose $m$ in a combination of the reference spectra. If we assume this to be linear, we have:

$$
m=\sum_{i=1}^{k} x_{i} s_{i}+r
$$

where $s_{i}$ is the fraction or abundance of the reference spectrum $i$ in $m$, and $r$ the residual vector. The latter is mostly composed by errors in the model and noise. The errors derive from contributions related to materials not present in $A$, subtle variations of one or more materials in $A$, noise affecting the selected reference spectra, and non-linear mixing effects. The noise mostly comes from fluctuations in the pixel values due to the low SNR in some bands caused by atmospheric absorption, and instrument-induced noise. If the modelling errors in $A$ are kept to a minimum, we expect the noise term to be predominant in the residual vector for bands with low SNR, and we can derive a reconstruction $\hat{m}$ as: 


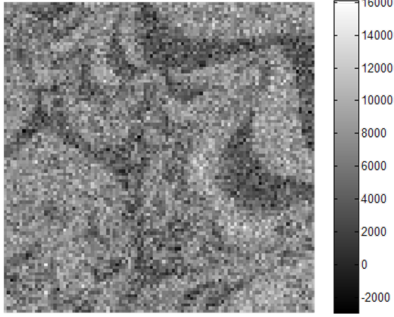

(a)

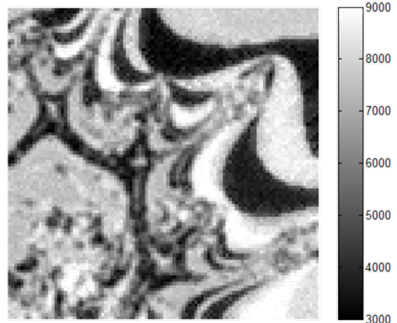

(c)

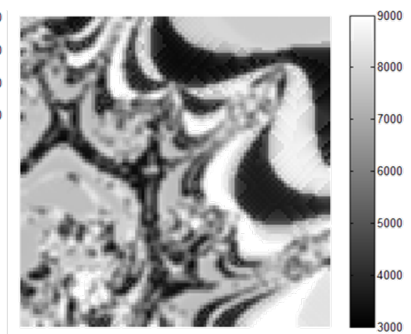

(b)

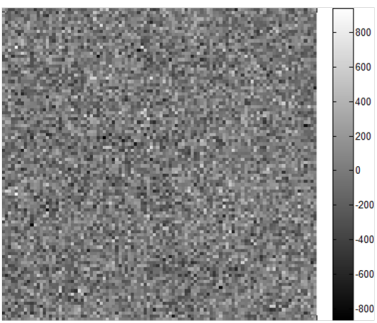

(d)
Figure 1: Results on synthetic dataset with perfect model available. From top left: (a) Band 40 from fractals synthetic dataset, heavily corrupted $(\mathrm{SNR}=10)$; (b) Band 40 without noise, ideal target; (c) Image (b) restored from (a) through UBD using the 9 original spectra; (d) Difference image obtained subtracting image (c) from image (a).

$$
\hat{m}=\sum_{i=1}^{k} x_{i} s_{i}
$$

ignoring $r$, and along with it most of the noise affecting $m$. The described procedure is based on the assumption that if the contributions to the radiation reflected from a resolution cell are known, the value of noisy bands in that area can be derived by a combination of the average values characterizing each component in that spectral range. The method needs as input a set of spectra that well characterize the scene, and is carried out independently for each pixel. As a certain homogeneity of the classes of interest is assumed, the method is expected to perform better on natural scenes where man-made objects (usually having a higher variability) are not prevalent.

\section{ITERATIVE REFERENCE SPECTRA SELECTION}

The results obtained with the UBD method as illustrated in section 2 are dependent on the quality of the linear mixing model used. This section illustrates which results can be expected in an ideal case, and what can be done to mitigate the distortions introduced by errors in the adopted model. Special attention is given on how to include in the model all relevant spectra useful to recover a given band in a scene. In the following experiments we choose Non-negative Least Squares (NNLS) as unmixing algorithm (Bioucas-Dias et al., 2012).

\subsection{An ideal case}

In an ideal case, we have a perfect model comprising all the spectra related to materials within the scene, all such spectra are noisefree and there are no subtle variations within a given material in terms of percentage of scattered energy at a given wavelength. To understand how the algorithm would work in such a case, we

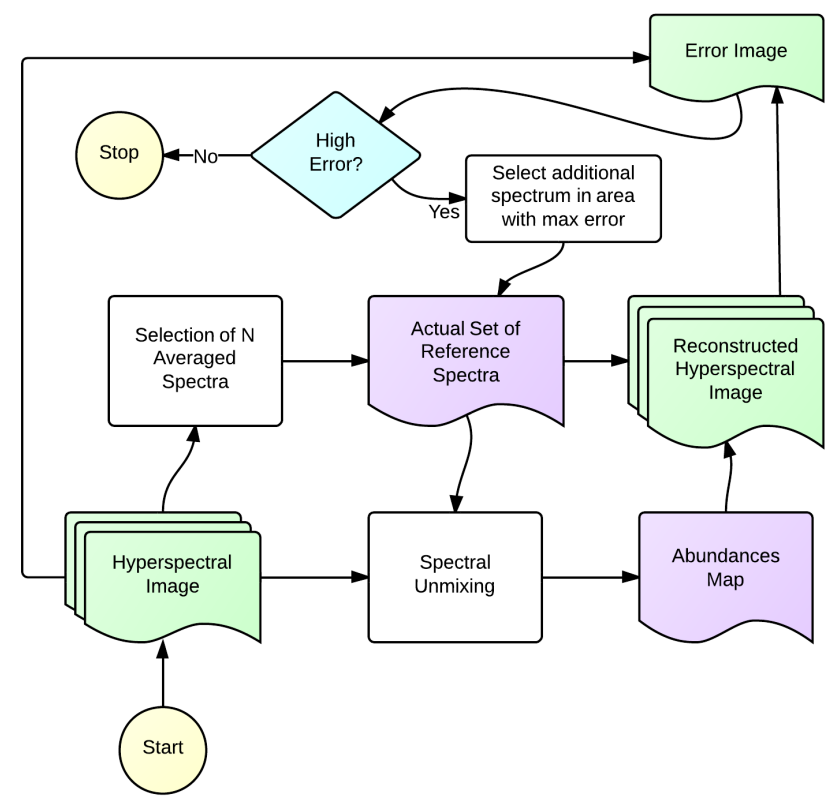

Figure 2: Workflow for the reported algorithm. The iterations update the set of reference spectra to be employed in the linear mixing model, keeping as much information as possible in a given noisy band and discarding all noise contributions.

apply UBD to a synthetic hyperspectral dataset by J. Plaza et al. (Plaza et al., 2012), available at (Plaza and Plaza, 2012). The dataset includes images composed by mixtures of 9 known pure spectral signatures with different noise levels (SNR ranging from 10 to $\infty$ ). The spectra are selected from the USGS spectral library (Clark et al., 2007), and the images are of size $100 \times 100$ pixels and have 221 bands between 0.4 and $2.5 \mu \mathrm{m}$.

We consider the image with the worse SNR which is 10 , and try to reconstruct the noise-free image from the noisy one, given the original noise-free spectra used to generate the target image, as in eq. 2. Results in fig. 1 show that, in spite of the high noise power, the target image is retrieved almost perfectly. As objective evaluation parameters we compute the average Normalized Root Mean Square Error (NRMSE) and the average Spectral Angle (SA) value, between the two images. The former is expressed in percentage as:

$$
\operatorname{NRMSE}(x, y)=\frac{\sqrt{\frac{\sum_{i=1}^{n}\left(x_{i}-y_{i}\right)^{2}}{n}}}{x_{\max }-x_{\min }},
$$

with $x_{\max }$ and $x_{\min }$ being the highest and lowest values assumed by $x$ respectively, and the numerator the root of the mean squared error over $n$ samples. The SA, which measures the angle between two vectors representing two spectra. It is defined as the arccosine of the dot-product between two vectors $x$ and $y$ as (Kruse, 1993):

$$
S A(x, y)=\cos ^{-1} \frac{\sum_{i=1}^{n} x_{i} y_{i}}{\sqrt{\sum_{i=1}^{n} x_{i}^{2}} \sqrt{\sum_{i=1}^{n} y_{i}^{2}}}
$$

In this case after reconstruction the NMSE is $2.4 \%$, while the average SA value is as low as 0.0346 . 

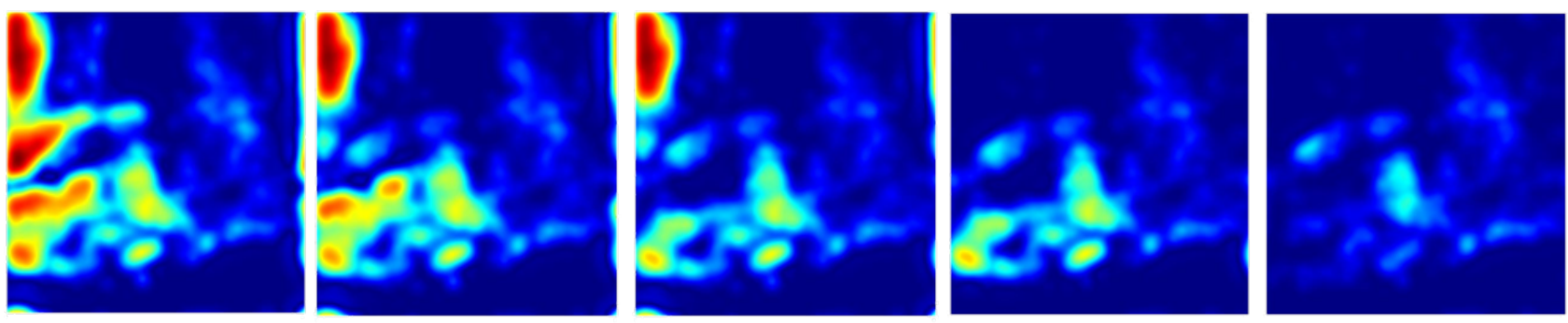

Figure 3: Sample steps from the iterative reference spectra selection. The reported images show the reconstruction error in a sample band at $434 \mathrm{~nm}$, after a low-pass filtering carried out in the frequency domain. Red and dark blue areas correspond to high errors and to errors close to 0 , respectively.

\subsection{Real cases}

In a real case the mixing model is generally unknown, along with the noise power and its distribution. Assuming non-linear mixing phenomenons to be negligible (Keshava and Mustard, 2002), the three main problems are: noisy reference spectra used as basis for the unmixing step, subtle variations of the same material which are not captured in the unmixing model, and missing spectra in the model. We briefly illustrate how to deal with these problems, with special attention to the last one.

The problem of noise presence in the basis used for the unmixing step can be strongly mitigated by considering each spectrum as the average of several similar signals. We assume the noise to be additive white Gaussian with zero mean, and signal-dependent noise to be negligible (Aiazzi et al., 2006). Whenever a spectrum is included into the model, the average values for each wavelength in a homogeneous area can be considered. This way, the mean of the noise in the considered spectra will be close to 0 , and the typical values for a spectrum in bands affected by low SNR will be reliable. If no such area can be found, the average spectrum can be computed using the spectra in the image which are the most similar to the initial one, and minimizes the SA between the two. Note that we are assuming that, for a given material, it is possible to identify several pixels in the image which are macroscopically pure up to a certain degree. This also implies that we are not looking specifically for pure materials or endmembers, as also intimate mixtures represent valid candidates.

About the subtle variations within each material, nothing can be done if these cannot be expressed as linear combinations of the reference spectra used as basis for the unmixing step. This is a limitation of the method but it can be solved by considering different samples for the same material, which can in turn be mixed to obtain several intermediate states for the spectral response of any image element. This is done under the assumption that adding a spectrum which only slightly differs from one already present in the model does not make the system unstable, i.e. does not cause one of the reference spectra to be linearly dependent from the others. This would introduce non-negligible numerical errors in the unmixing step, which requires the inversion of the matrix composed by the reference spectra.

This section mainly deals with the last of the described problems: finding all the spectra in the image which are needed to reconstruct an image discarding only its random noise component but keeping all relevant information in bands affected by low SNR. We could use any endmember extraction algorithm to retrieve the spectra which can at best represent the contents of a scene, but these would be driven by the full spectral information of a given image element, and would therefore implicitly give less importance to variations in bands with low SNR. As these are exactly the ones we want to retrieve, traditional endmember extraction algorithms are not fit to derive a model focused at keeping all the relevant information in these bands. Instead, we propose to use the following algorithm to retrieve the reference spectra which are useful to reconstruct one of such bands, for which the workflow is sketched in fig. 2.

First of all, the set of reference spectra must be initialized. For this purpose, traditional algorithms which perform a rotation of the original data in a hyperplane with orthogonal components can be applied, such as Principal Components Analysis (PCA) (Kaewpijit et al., 2003) or Minimum Noise Fraction (MNF) (Amato et al., 2009) can be used. Selecting the extreme points in the space spanned by the first two dimensions in such spaces makes sure that the spectral information they convey is as uncorrelated as possible. In this way, 4 spectra can be selected to form the starting model for the unmixing algorithm.

Afterwards, an iterative reference spectra refinement method is carried out. In each step, the UBD algorithm is applied with the current mixing model, yielding a reconstructed image which will have at first high distortions with respect to the original one. At this point, the noisy band which is useful for a given application is selected, and an error image is generated by subtracting it from the original noisy band. The resulting error image will be composed by random noise and diffuse errors which are linked to the relevant information we lost by representing the image as a linear mixture of too few reference spectra. To separate these two components, the error image is filtered in the frequency domain with a Butterworth low-pass filter, yielding an error image containing only errors which are diffuse over an area. A filtered error image is of the kind reported in fig. 3. At this point, a new spectrum is selected from the area with maximum error and averaged over its neighbours (or similar pixels if the area is not homogeneous) to reduce noise contributions. The spectrum is added to the model and a new iteration takes place. As new spectra are added to the model, the error in the reconstructed image decreases: fig. 3 shows an example of the different outcomes of several iterations on the band at $434 \mathrm{~nm}$ from a hyperspectral scene acquired by the HySpex sensor over the lake Starnberg in Germany. The original band is reported along with its final denoised version in figs. 4 and 5. In the processing the land and part of the boats have been masked out for computational efficiency reasons, as the main motivation for denoising this particular band is its utility in estimating the concentration of Coloured dissolved organic matter (CDOM) in natural waters, which is often carried out using information at different wavelengths, given the low SNR of some bands in the blue portion of the spectrum (Kutser et al., 2005). After applying UBD, the retrieved CDOM parameters are closer to their actual values (Cerra et al., 2013a). 


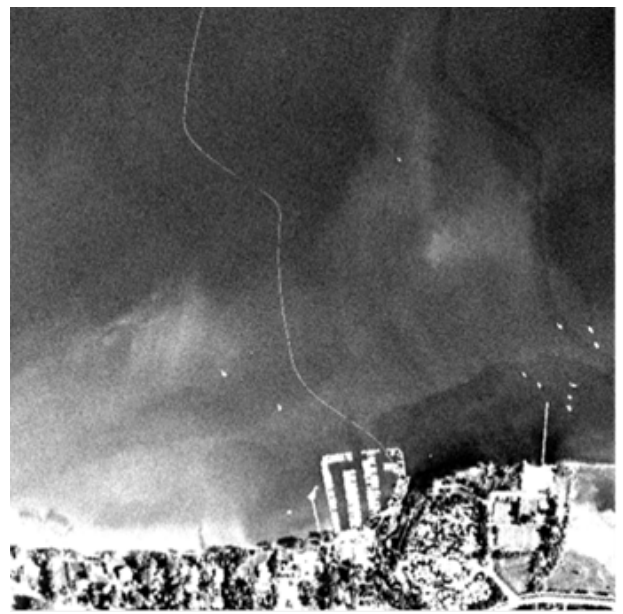

Figure 4: Band 6 from the Starnberger lake dataset (434 nm).

\section{CONCLUSIONS AND FUTURE WORK}

Unmixing-based Denoising (UBD) is a supervised methodology for the recovery of bands characterized by a low Signal-to-Noise Ratio (SNR) in a hyperspectral scene. UBD reconstructs any pixel in a given band as a linear combination of reference spectra belonging to materials present in the scene. As the residual vector from the unmixing process is mostly composed by contributions of uninteresting materials, unwanted atmospheric influences and sensor-induced noise, this is ignored in the reconstruction process.

This paper focuses on how to include in the linear mixing model all relevant reference spectra to maximize the information which is kept in a given spectral band. The main problem when adopting this approach is deciding the stop criterion. In this work a threshold has been set as the maximum value which is allowed for an error image after a low-pass filtering in frequency, but several different criteria could be chosen. For example, spectra could be added until they are linearly independent regardless of the highest value in the error image. Or the virtual dimensionality of the dataset could be estimated (Bioucas-Dias and Nascimento, 2008), and the process could stop when the same number of reference spectra is collected.

The proposed method could be applied to retrieve important information in noisy bands for a wide array of practical applications. Examples include: estimation of Leaf Clorophyll Content and vegetation stress for given kinds of crops, estimation of oil thickness in oil spill applications, estimation of CDOM in open waters, and vegetation damage severity index after forest fires.

\section{REFERENCES}

Aiazzi, B., Alparone, L., Barducci, A., Baronti, S., Marcoionni, P., Pippi, I. and Selva, M., 2006. Noise modelling and estimation of hyperspectral data from airborne imaging spectrometers. Annals of Geophysics.

Amato, U., Cavalli, R. M., Palombo, A., Pignatti, S. and Santini, F., 2009. Experimental approach to the selection of the components in the minimum noise fraction. IEEE Transactions on Geoscience and Remote Sensing 47(1-1), pp. 153-160.

Bioucas-Dias, J. and Nascimento, J., 2008. Hyperspectral subspace identification. IEEE Transactions on Geoscience and Remote Sensing 46(8), pp. $2435-2445$.

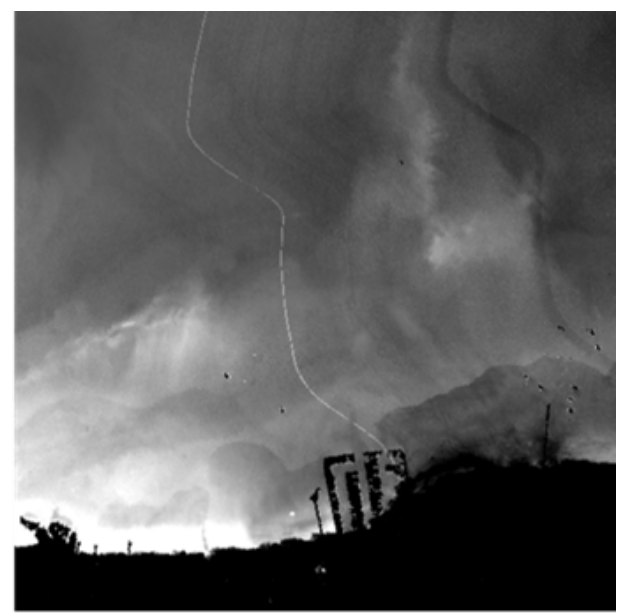

Figure 5: Image in fig. 4 after denoising. Land and boats have been masked out.

Bioucas-Dias, J. M., Plaza, A., Dobigeon, N., Parente, M., Du, Q., Gader, P. and Chanussot, J., 2012. Hyperspectral unmixing overview: Geometrical, statistical, and sparse regression-based approaches. IEEE Journal of Selected Topics in Applied Earth Observations and Remote Sensing 5(2), pp. 354-379.

Cerra, D., Müller, R. and Reinartz, P., 2013a. Exploiting noisy hyperspectral bands for water analysis. In: Proc. 33rd EARSeL Symposium.

Cerra, D., Müller, R. and Reinartz, P., 2013b. Noise reduction in hyperspectral images through spectral unmixing. Geoscience and Remote Sensing Letters, IEEE PP(99), pp. 1-1.

Clark, R., Swayze, G., Wise, R., Livo, E., Hoefen, T., Kokaly, R. and Sutley, S., 2007. Usgs digital spectral library splib06a: U.s. geological survey. Digital Data Series.

Kaewpijit, S., Le Moigne, J. and El-Ghazawi, T., 2003. Automatic reduction of hyperspectral imagery using wavelet spectral analysis. IEEE Transactions on Geoscience and Remote Sensing 41(4), pp. $863-871$.

Keshava, N. and Mustard, J., 2002. Spectral unmixing. IEEE Signal Processing Magazine 19(1), pp. 44 -57.

Kruse, F., 1993. The Spectral Image Processing System (SIPS) - Interactive Visualization and Analysis of Imaging Spectrometer Data. Remote Sensing of Environment 44, pp. 145-163.

Kutser, T., Pierson, D. C., Kallio, K. Y., Reinart, A. and Sobek, S., 2005. Mapping lake cdom by satellite remote sensing. Remote Sensing of Environment 94(4), pp. 535 - 540.

Plaza, J. and Plaza, A., $2012 . \quad$ Hypermix. http://www.hypercomp.es/hypermix.

Plaza, J., Hendrix, E. M. T., Garca, I., Martin, G. and Plaza, A., 2012. On endmember identification in hyperspectral images without pure pixels: A comparison of algorithms. Journal of Mathematical Imaging and Vision 42(2-3), pp. 163-175.

Renard, N., Bourennane, S. and Blanc-Talon, J., 2008. Denoising and dimensionality reduction using multilinear tools for hyperspectral images. IEEE Geoscience and Remote Sensing Letters $5(2)$, pp. $138-142$. 